\title{
NONDECREASING LOWER BOUND ON THE POISSON CUMULATIVE DISTRIBUTION FUNCTION FOR $z$ STANDARD DEVIATIONS ABOVE THE MEAN
}

\author{
M. BONDAREVA, ${ }^{*}$ University of Rochester
}

\begin{abstract}
In this paper we discuss a nondecreasing lower bound for the Poisson cumulative distribution function (CDF) at $z$ standard deviations above the mean $\lambda$, where $z$ and $\lambda$ are parameters. This is important because the normal distribution as an approximation for the Poisson CDF may overestimate or underestimate its value. A sharp nondecreasing lower bound in the form of a step function is constructed. As a corollary of the bound's properties, for a given percent $\alpha$ and parameter $\lambda$, the minimal $z$ is obtained such that, for any Poisson random variable with the mean greater or equal to $\lambda$, its $\alpha$ th percentile is at most $z$ standard deviations above its mean. For Poisson distributed control parameters, the corollary allows simple policies measuring performance in terms of standard deviations from a benchmark.
\end{abstract}

Keywords: Central limit theorem; Poisson distribution; monotonic approximation

2010 Mathematics Subject Classification: Primary 60E05

\section{Introduction}

In this paper we study a nondecreasing lower bound for the Poisson cumulative distribution function $(\mathrm{CDF})$ at $z$ standard deviations above the mean. Management widely uses policies that measure performance in terms of standard deviations from a benchmark. A common assumption is that a random control parameter is normally distributed. For normal random variables, the $\mathrm{CDF}$ at $z$ standard deviations above the mean is well known. This is not the case if the control parameter instead has a Poisson distribution. Let $X_{\lambda}$ be a Poisson random variable with mean $\lambda \geq 0$. For large values of $\lambda$, the normal distribution with mean $\lambda$ and variance $\lambda$ is a good approximation to the Poisson distribution. Nevertheless, the normal CDF may underestimate or overestimate the Poisson CDF. This is unacceptable for cases with high penalties not meeting the target values. The lower bound on the Poisson CDF given in this paper solves the problem.

This situation arises in the following setting. Suppose that a store faces Poisson demand and the owner would like to satisfy it at least $95 \%$ of the time. The demand rate $\lambda$ is random and will be known later. Currently, the owner would like to know what his/her expected inventory cost will be. Let $G$ be the prior distribution of $\lambda$. One approach is to calculate the 95th percentile for the Poisson distribution for every possible realization of $\lambda$ and compute the expectation using $G$. This approach is difficult, because the question of defining a closed-form solution for the percentiles of the Poisson distribution with an arbitrary mean is still unresolved. Another approach is to determine the constant $z$ based on the support of $G$ such that $\lambda+z \sqrt{\lambda}$ satisfies demand at least $95 \%$ of the time, and then calculate the expected value of $\lambda+z \sqrt{\lambda}$. This will be simpler and will yield an analytical expression. This is the result given in Corollary 1 below.

Received 5 May 2011; revision received 13 December 2012.

* Postal address: William E. Simon Graduate School of Business Administration, University of Rochester, Rochester, NY 14627, USA. Email address: mariya.bondareva@simon.rochester.edu 
Let the probability that $X_{\lambda}$ is not greater than $z$ standard deviations above its mean be $\Phi_{P}(\lambda, z)=\mathbb{P}\left(X_{\lambda} \leq \lambda+z \sqrt{\lambda}\right), z \geq 0$. As will be shown below, there exists a step function $\Phi_{P}(\lambda, z)$ such that, for all $\lambda \geq 0$ and $z \geq 0, \Phi_{P}(\lambda, z)$ is a nondecreasing lower bound on $\overline{\Phi_{P}}(\lambda, z)$. In addition, $\Phi_{P}(\lambda, z)$ is the unique sharp lower bound among all nondecreasing step functions. As an interesting corollary of the properties of $\Phi_{P}(\lambda, z)$, for any given percent $\alpha$ and parameter $\lambda$, the minimal $z$ is obtained such that, for any Poisson random variable with the mean greater or equal to $\lambda$, its $\alpha$ th percentile is at most $z$ standard deviations above its mean.

Theorem 1 in this paper generalizes Theorem 2.3 of [2]. The nondecreasing property of $\Phi_{P}(\lambda, z)$ extends the conditions for the Poisson distribution under which the monotonic approach to central limits holds.

In Section 2 we give the proofs of the theorem and the corollary. In Section 3 we discuss the results and give one application.

\section{Lower bound on $\Phi_{P}(\lambda, z), z \geq 0$}

Because $X_{\lambda}$ takes integer values, it is clear that

$$
\Phi_{P}(\lambda, z)=\mathbb{P}\left(X_{\lambda} \leq\lfloor\lambda+z \sqrt{\lambda}\rfloor\right)=\sum_{i=0}^{\lfloor\lambda+z \sqrt{\lambda}\rfloor} \frac{\mathrm{e}^{-\lambda} \lambda^{i}}{i !} .
$$

Given $z, \lambda_{n}(z)$ is defined as the value of $\lambda$ such that $\lambda+z \sqrt{\lambda}=n$. For a fixed $z, \Phi_{P}(\lambda, z)$ is a right-continuous function with jumps at $\lambda_{n}(z)$. In Lemma 1 below we examine $\lambda_{n}(z)$ and the discontinuity of $\Phi_{P}(\lambda, z)$. Figure 1 shows $\Phi_{P}(\lambda, 0.5)$ and $\Phi_{P}(\lambda, 2)$.

Note that $X_{\lambda}$ is the number of arrivals in the interval $(0, \lambda]$ for a rate-one stochastic Poisson process. Let $S_{n}$ be the time of the $n$th Poisson event, and let $S_{0}=0$. Then $S_{n}=\sum_{i=1}^{n} T_{i}$, where $T_{i}$ are independent, exponentially distributed random variables with mean 1 . Throughout this paper, $\mathbb{N}$ denotes the set of positive integers, i.e. $\mathbb{N}=\{1,2, \ldots\}$. For $n \in \mathbb{N}, \mathbb{P}\left(X_{\lambda} \leq n-1\right)=$ $\mathbb{P}\left(S_{n}>\lambda\right)$, so

$$
\Phi_{P}(\lambda, z)=\mathbb{P}\left(S_{\lfloor\lambda+z \sqrt{\lambda}\rfloor+1}>\lambda\right) .
$$

Lemma 1. Let $\left(\lambda_{n}(z): n \in \mathbb{N}\right)$ be the sequence of positive-real functions defined by $\lambda_{n}(z)=$ $\left(\sqrt{(z / 2)^{2}+n}-z / 2\right)^{2}, z \geq 0$. Let $\lambda_{0}(z)=0$ for all $z \geq 0$. Then $\Phi_{P}(\lambda, z)$ has positive jumps at $\lambda_{n}(z)$ and is decreasing in $\lambda$ in every interval $\left[\lambda_{n-1}(z) ; \lambda_{n}(z)\right)$. The functions $\lambda_{n}(z)$ are decreasing in $z$ and $\Phi_{P}\left(\lambda_{n}(z), z\right)$ is increasing in $z$.

Proof of Lemma 1. Fix $z \geq 0$, and consider the increasing concave function $\lambda+z \sqrt{\lambda}$ with the increasing convex inverse $\left(\sqrt{(z / 2)^{2}+y}-z / 2\right)^{2}$, where $\lambda \geq 0$ and $y \geq 0$. Clearly, $\lambda_{n}(z)$ is the value of the inverse at $y=n$. Then, from (1), $\Phi_{P}(\lambda, z)$ has jumps at $\lambda_{n}(z)$ and, from (2), $\Phi_{P}(\lambda, z)$ decreases in $\lambda$ for $\lambda \in\left[\lambda_{n}(z) ; \lambda_{n+1}(z)\right)$.

Since $\sqrt{(z / 2)^{2}+n}-z / 2=n /\left(\sqrt{z^{2} / 4+n}+z / 2\right)$ is a decreasing function of $z, \lambda_{n}(z)$ decreases in $z$. If $0 \leq z_{1}<z_{2}$ then

$$
\Phi_{P}\left(\lambda_{n}\left(z_{1}\right), z_{1}\right)=\mathbb{P}\left(S_{n+1}>\lambda_{n}\left(z_{1}\right)\right)<\mathbb{P}\left(S_{n+1}>\lambda_{n}\left(z_{2}\right)\right)=\Phi_{P}\left(\lambda_{n}\left(z_{2}\right), z_{2}\right) .
$$

This completes the proof.

The step function $\Phi_{P}(\lambda, z)$ constructed below is a lower bound on $\Phi_{P}(\lambda, z)$. For $z \geq 0$, define

$$
\underline{\Phi_{P}}(\lambda, z)=\mathbb{P}\left(X_{\lambda_{n}(z)} \leq n-1\right), \quad \lambda \in\left[\lambda_{n-1}(z) ; \lambda_{n}(z)\right), n \in \mathbb{N} .
$$

As an example, we present the graph of $\Phi_{P}(\lambda, 0.5)$ in Figure 1. 


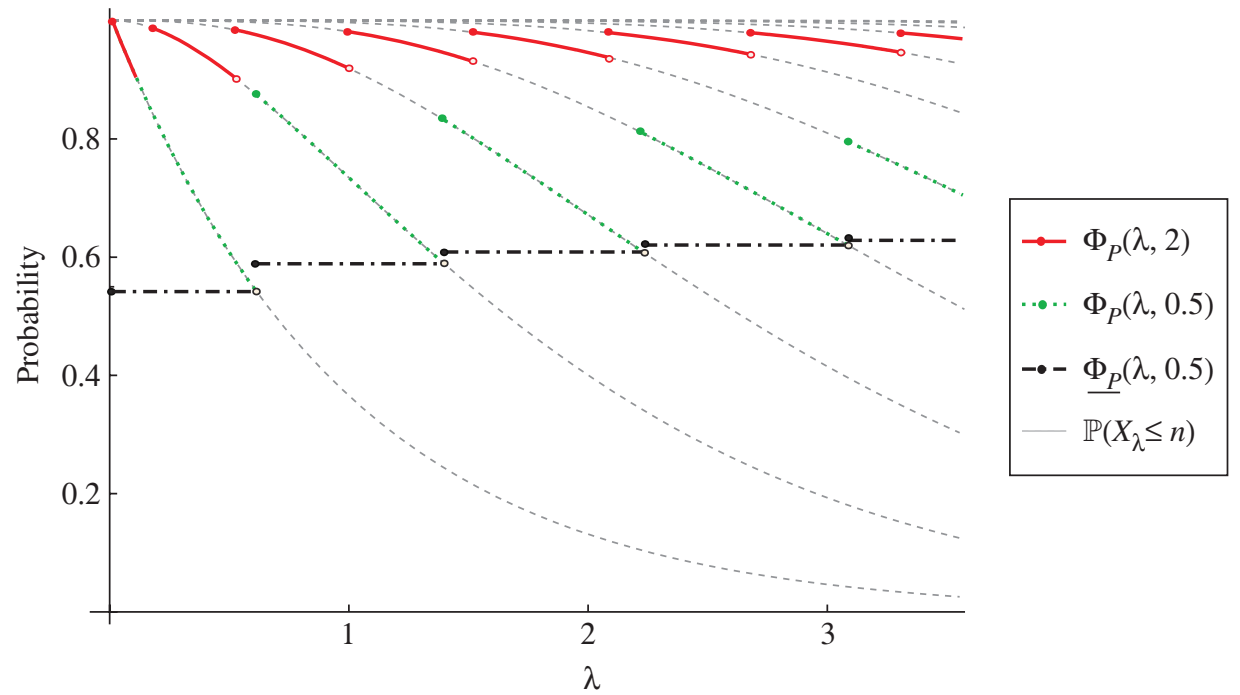

FiguRE 1: $\Phi_{P}(\lambda, 2), \Phi_{P}(\lambda, 0.5), \underline{\Phi_{P}}(\lambda, 0.5)$, and $\mathbb{P}\left(X_{\lambda} \leq n\right)$ for $n \in\{0,1, \ldots, 10\}$.

In order to prove that $\Phi_{P}(\lambda, z)$ is bounded below by $\underline{\Phi_{P}}(\lambda, z)$, note that $\Phi_{P}(\lambda, z)=$ $\lim _{\mu \rightarrow \lambda_{n}(z)-} \Phi_{P}(\mu, z)$ for $\lambda \in\left[\lambda_{n-1}(z) ; \lambda_{n}(z)\right)$, and that, from Lemma $1, \Phi_{P}(\lambda, \bar{z})$ is decreasing in $\lambda$. As a result, for $\lambda \in\left[\lambda_{n-1}(z) ; \lambda_{n}(z)\right)$,

$$
\Phi_{P}(\lambda, z)>\lim _{\mu \rightarrow \lambda_{n}(z)-} \Phi_{P}(\mu, z)=\underline{\Phi_{P}}(\lambda, z) .
$$

From (4) and Lemma $1, \Phi_{P}(\lambda, z)$ is a lower bound of $\Phi_{P}(\lambda, z)$, is right continuous, and has jumps at $\lambda_{n}(z)$. Note that the left-continuous version of $\underline{\Phi_{P}}(\lambda, z)$ would be inferior to the right-continuous version at $\lambda_{n}(z), n \in \mathbb{N}$.

Using Lemma 1, Theorem 1 below proves nondecreasing properties of $\underline{\Phi_{P}}(\lambda, z)$ and its convergence to the standard normal CDF.

Theorem 1. (a) For $\lambda \geq 0$ and $z \geq 0, \underline{\Phi_{P}}(\lambda, z)$ is nondecreasing in $\lambda$ and increasing in $z$.

(b) For $z \geq 0, \lim _{\lambda \rightarrow \infty} \underline{\Phi_{P}}(\lambda, z)=\Phi(z)$, where $\Phi(z)$ is the standard normal CDF.

Proof of Theorem 1. (a) To prove that $\Phi_{P}(\lambda, z)$ is nondecreasing in $\lambda$, it is enough to show that its jumps at $\lambda_{n}(z)$ are positive for every nonnegative $z$. Let $\delta(n, z)$ denote the size of a jump of the step function $\underline{\Phi_{P}}(\lambda, z)$ at $\lambda=\lambda_{n}$, that is, $\delta(n, z)=\underline{\Phi_{P}}\left(\lambda_{n}(z), z\right)-\underline{\Phi_{P}}\left(\lambda_{n-1}(z), z\right)$, $n \in \mathbb{N}$.

First, consider $z=0$. Note that $\delta(n, 0)=\mathbb{P}\left(S_{n+1} \geq n+1\right)-\mathbb{P}\left(S_{n} \geq n\right)$, where $S_{n}=$ $\sum_{i=1}^{n} T_{i}$. From Theorem 2.2 of [2], $\mathbb{P}\left(\sum_{i=1}^{n} T_{i} \geq n\right)$ is a strictly increasing function of $n$. Therefore, $\delta(n, 0)>0$.

Second, consider $z>0$. Noting that $\delta(n, z)=\mathbb{P}\left(X_{\lambda_{n+1}} \leq n\right)-\mathbb{P}\left(X_{\lambda_{n}} \leq n-1\right)$ and recalling the Poisson-gamma relation as in Equation (4) of [1], it follows that

$$
\delta(n, z)=\frac{1}{n !} \int_{\lambda_{n+1}(z)}^{\infty} u^{n} \mathrm{e}^{-u} \mathrm{~d} u-\frac{1}{(n-1) !} \int_{\lambda_{n}(z)}^{\infty} u^{n-1} \mathrm{e}^{-u} \mathrm{~d} u .
$$

To simplify the notation, define $\tilde{\delta}(n, z)=\delta(n, 2 z)$. To show that $\delta(n, z)>0$ for all $z>0$ and $n \in \mathbb{N}$, it is enough to establish that $\tilde{\delta}(n, z)$ is positive for all $z>0$ and $n \in \mathbb{N}$. Fix $n \in \mathbb{N}$. 
Clearly, $\tilde{\delta}(n, 0)=\delta(n, 0)>0$. Below it is proved that there exists a positive $\tilde{z}$ such that $\tilde{\delta}(n, z)$ increases in $z$ over $(0 ; \tilde{z}]$ and asymptotically decreases to 0 over $(\tilde{z} ; \infty)$.

To analyze $\tilde{\delta}(n, z)$, define the following functions:

$$
\begin{aligned}
& h(n, z)=\mathrm{e}^{-\left(\sqrt{z^{2}+n}-z\right)^{2}} \frac{\left(\sqrt{z^{2}+n}-z\right)^{2 n}}{\sqrt{z^{2}+n}}, \\
& w(n, z)=n \frac{h(n, z)}{h(n+1, z)}, \\
& b(n, z)=4 z \sqrt{z^{2}+n} \sqrt{z^{2}+n+1}\left(\sqrt{z^{2}+n+1}-\sqrt{z^{2}+n}\right)-1, \quad n \in \mathbb{N} .
\end{aligned}
$$

Then,

$$
\frac{\partial \tilde{\delta}(n, z)}{\partial z}=\frac{2}{n !} h(n+1, z)(1-w(n, z)) .
$$

Since $h(n, z)$ is positive for $n \in \mathbb{N}$, the sign of $\partial \tilde{\delta}(n, z) / \partial z$ is that of $(1-w(n, z))$. To characterize $w(n, z)$, consider its derivative

$$
\frac{\partial w(n, z)}{\partial z}=n z \frac{h(n, z) b(n, z)}{h(n+1, z)\left(z^{2}+n\right)\left(z^{2}+n+1\right)} .
$$

The sign of $b(n, z)$ determines the sign of $\partial w(n, z) / \partial z$. Note that $b(n, 0)=-1$,

$$
\lim _{z \rightarrow \infty} b(n, z)=\infty
$$

and

$$
\begin{aligned}
\frac{\partial b(n, z)}{\partial z}= & 4 z^{2}\left(\sqrt{z^{2}+n+1}-\sqrt{z^{2}+n}\right) \\
& \times\left(\frac{\sqrt{\left(z^{2}+n\right)\left(z^{2}+n+1\right)}}{z^{2}}+\frac{\sqrt{z^{2}+n}}{\sqrt{z^{2}+n+1}}+\frac{\sqrt{z^{2}+n+1}}{\sqrt{z^{2}+n}}-1\right) .
\end{aligned}
$$

Clearly, $\partial b(n, z) / \partial z>0$. As a result, $b(n, z)$ increases strictly monotonically in $z$ and changes sign from negative to positive at some $z^{*}$ that depends on $n$. Then, from (7), $w(n, z)$ decreases in $z$ over $\left(0 ; z^{*}\right]$ and increases over $\left(z^{*} ; \infty\right)$. According to the inequality proved by Khattri [3], $w(n, 0)=\mathrm{e}(n /(1+n))^{1 / 2+n}<1$ for all $n \in \mathbb{N}$. In addition, $\lim _{z \rightarrow \infty} w(n, z)=\infty$. Therefore, there exists a $\tilde{z}$ depending on $n$ such that $\tilde{z}>z^{*}$ and $w(n, \tilde{z})=1$. If $z \in(0 ; \tilde{z})$ then $w(n, z)<1$ and if $z \in(\tilde{z} ; \infty)$ then $w(n, z)>1$.

Consequently, from $(6), \tilde{\delta}(n, z)$ increases in $z$ over $(0 ; \tilde{z}]$ and decreases over $(\tilde{z} ; \infty)$. It was proved that $\tilde{\delta}(n, 0)>0$. Also, $\lim _{z \rightarrow \infty} \tilde{\delta}(n, z)=0$ because both integrals in (5) converge to 0 . It follows, for all $z \geq 0$, that $\tilde{\delta}(n, z)>0$. Therefore, for all $z>0$ and $n \in \mathbb{N}, \delta(n, z)>0$ and the function $\Phi_{P}(\lambda, z)$ is nondecreasing in $\lambda$.

To prove that $\underline{\Phi_{P}}(\lambda, z)$ increases in $z$, note that, for $\lambda \in\left[\lambda_{n-1}(z) ; \lambda_{n}(z)\right)$,

$$
\underline{\Phi_{P}}(\lambda, z)=\lim _{\mu \rightarrow \lambda_{n}(z)-} \Phi_{P}(\mu, z)=\Phi_{P}\left(\lambda_{n}(z), z\right)-\frac{\mathrm{e}^{-\lambda_{n}(z)}\left(\lambda_{n}(z)\right)^{n}}{n !}, \quad n \in \mathbb{N} .
$$

For arbitrarily chosen $z_{1}$ and $z_{2}$ such that $0 \leq z_{1}<z_{2}$, any positive $\lambda$ belongs to the two intervals $\lambda \in\left[\lambda_{i-1}\left(z_{1}\right) ; \lambda_{i}\left(z_{1}\right)\right)$ and $\lambda \in\left[\lambda_{j-1}\left(z_{2}\right) ; \lambda_{j}\left(z_{2}\right)\right)$, where $i \in \mathbb{N}$ and $j \in \mathbb{N}$. From Lemma $1, \lambda_{n}(z)$ decreases in $z$. Therefore, $j \geq i$ and $\lambda_{i}\left(z_{2}\right)<\lambda_{i}\left(z_{1}\right)$. 
As proved in Lemma $1, \Phi_{P}\left(\lambda_{i}\left(z_{1}\right), z_{1}\right)<\Phi_{P}\left(\lambda_{i}\left(z_{2}\right), z_{2}\right)$. Note that, for $\lambda \in[0 ; i]$, the function $\mathrm{e}^{-\lambda} \lambda^{i}$ is increasing in $\lambda$. Recalling that $\lambda_{n}(z)$ is defined as the value of $\lambda$ such that $\lambda+z \sqrt{\lambda}=n$, we conclude that $\lambda_{i}\left(z_{2}\right)<\lambda_{i}\left(z_{1}\right) \leq i$. Then $\mathrm{e}^{-\lambda_{i}\left(z_{2}\right)} \lambda_{i}\left(z_{2}\right)^{i}<\mathrm{e}^{-\lambda_{i}\left(z_{1}\right)} \lambda_{i}\left(z_{1}\right)^{i}$. As a result,

$$
\begin{aligned}
\underline{\Phi_{P}}\left(\lambda, z_{1}\right) & =\Phi_{P}\left(\lambda_{i}\left(z_{1}\right), z_{1}\right)-\frac{\mathrm{e}^{-\lambda_{i}\left(z_{1}\right)}\left(\lambda_{i}\left(z_{1}\right)\right)^{i}}{i !} \\
& <\Phi_{P}\left(\lambda_{i}\left(z_{2}\right), z_{2}\right)-\frac{\mathrm{e}^{-\lambda_{i}\left(z_{2}\right)}\left(\lambda_{i}\left(z_{2}\right)\right)^{i}}{i !} \\
& =\underline{\Phi_{P}}\left(\lambda_{i-1}\left(z_{2}\right), z_{2}\right) .
\end{aligned}
$$

Since $j \geq i$, then $\lambda_{i-1}\left(z_{2}\right) \leq \lambda_{j-1}\left(z_{2}\right)$. By (8) and the fact that $\underline{\Phi_{P}}(\lambda, z)$ is nondecreasing in $\lambda$,

$$
\underline{\Phi_{P}}\left(\lambda, z_{1}\right)<\underline{\Phi_{P}}\left(\lambda_{i-1}\left(z_{2}\right), z_{2}\right) \leq \underline{\Phi_{P}}\left(\lambda_{j-1}\left(z_{2}\right), z_{2}\right)=\underline{\Phi_{P}}\left(\lambda, z_{2}\right) .
$$

(b) From the definition in (3), for $z \geq 0$ and $\lambda \in\left[\lambda_{n-1}(z) ; \lambda_{n}(z)\right), n \in \mathbb{N}$,

$$
\begin{aligned}
\underline{\Phi_{P}}(\lambda, z) & =\mathbb{P}\left(X_{\lambda_{n}(z)} \leq n-1\right) \\
& =\mathbb{P}\left(X_{\lambda_{n}(z)} \leq \lambda_{n}(z)+z \sqrt{\lambda_{n}(z)}-1\right) \\
& =\mathbb{P}\left(\frac{X_{\lambda_{n}(z)}-\lambda_{n}(z)}{\sqrt{\lambda_{n}(z)}} \leq z-\frac{1}{\sqrt{\lambda_{n}(z)}}\right) .
\end{aligned}
$$

Since, as $\lambda \rightarrow \infty, \lambda_{n} \rightarrow \infty$ and $1 /\left(\sqrt{\lambda_{n}(z)}\right) \rightarrow 0$, then, by the central limit theorem, $\underline{\Phi_{P}}(\lambda, z) \rightarrow \Phi(z)$ as $\lambda \rightarrow \infty$. This completes the proof of the theorem.

The function $\Phi_{P}(\lambda, z)$ is a unique sharp lower bound among all nondecreasing step functions because $\Phi_{P}(\lambda, \bar{z})=\lim _{\mu \rightarrow \lambda_{n}(z)-} \Phi_{P}(\mu, z)$ and the left-continuous version of $\Phi_{P}(\lambda, z)$ is inferior to the right-continuous version at $\lambda_{n}(z), n \in \mathbb{N}$.

In Corollary 1 we examine $z(\lambda, \alpha)$, which is defined as the minimal $z$ such that, for any Poisson random variable with mean greater than or equal to $\lambda$, its $\alpha$ th percentile is at most $z$ standard deviations above its mean. That is, for $\lambda \geq 0$ and $\alpha \in[0 ; 1]$,

$$
z(\lambda, \alpha)=\min \left\{y: \mathbb{P}\left(X_{\mu} \leq \mu+y \sqrt{\mu}\right) \geq \alpha \text {, for all } \mu \geq \lambda \text { and } y \geq 0\right\} .
$$

Corollary 1. For $\lambda \geq 0, z \geq 0, \mathrm{e}^{-1} \leq \alpha<1$, and $n \in \mathbb{N}$, the following statements hold.

(a) $\mathbb{P}\left(X_{\lambda} \leq \lambda+z \sqrt{\lambda}\right) \geq \mathrm{e}^{-1}$.

(b) If $\mathbb{P}\left(X_{\lambda} \leq n-2\right)<\alpha \leq \mathbb{P}\left(X_{\lambda} \leq n-1\right)$ then $z(\lambda, \alpha)=(n-\mu) / \sqrt{\mu}$, where $\mu$ can be found numerically from $\mathbb{P}\left(X_{\mu} \leq n-1\right)=\alpha$.

(c) The $\alpha$ th percentile of a Poisson distribution is at most $(1+\log \alpha) /(\sqrt{-\log \alpha})$ standard deviations above its mean.

Proof of Corollary 1. (a) From (3), $\Phi_{P}(0,0)=\mathrm{e}^{-1}$. By Theorem 1(a), $\Phi_{P}(\lambda, z)$ is nondecreasing both in $\lambda$ and $z$. Then, for $\overline{\lambda \geq 0}$ and $z \geq 0, \underline{\Phi_{P}}(\lambda, z) \geq \underline{\Phi_{P}}(0, \overline{0)}$ and the result follows.

(b) Let $A=\left(\lambda_{A}, \alpha_{A}\right)$ with $\lambda_{A} \geq 0$ and $\alpha_{A} \in\left[\mathrm{e}^{-1} ; 1\right]$. The value $z\left(\lambda_{A}, \alpha_{A}\right)$ is found by the construction of the lower bound $\Phi_{P}\left(\lambda, z_{A}\right)$ going through $A$. Figure 2 shows the process. The curves $C_{i}$ represent $\mathbb{P}\left(X_{\lambda} \leq i\right), \overline{i \in}\{-1,0,1, \ldots\}$. Thus, $C_{-1}=0$.

First, find curves $C_{n-2}$ and $C_{n-1}$ such that point $A$ lies between them or belongs to $C_{n-1}$. That is, find $n$ such that $\mathbb{P}\left(X_{\lambda_{A}} \leq n-2\right)<\alpha_{A} \leq \mathbb{P}\left(X_{\lambda_{A}} \leq n-1\right)$. Then there exists $A^{+}=\left(\lambda_{A}^{+}, \alpha_{A}\right)$ 


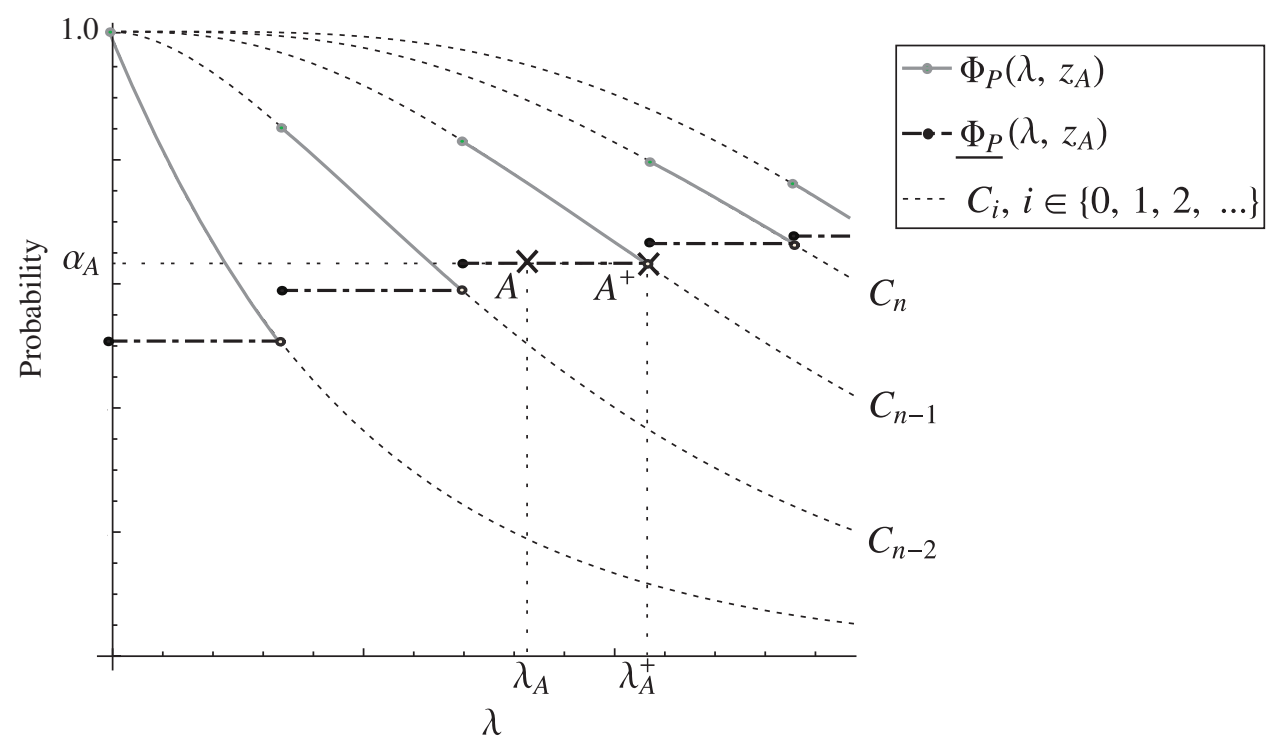

Figure 2: The construction of the lower bound $\underline{\Phi_{P}}\left(\mu, z_{A}\right)$, where $z_{A}=z\left(\lambda_{A}, \alpha_{A}\right)$. Points $A=\left(\lambda_{A}, \alpha_{A}\right)$ and $A^{+}=\left(\lambda_{A}^{+}, \alpha_{A}\right)$ are marked and the curves represent $C_{i}=\mathbb{P}\left(X_{\lambda} \leq i\right), i \in\{-1,0,1, \ldots\}$.

such that $A^{+}$belongs to curve $C_{n-1}$. The value $\lambda_{A}^{+}$can be found by numerically solving for $\mu$ in the equation $\mathbb{P}\left(X_{\mu} \leq n-1\right)=\alpha_{A}$. Then, choose $z_{A}$ so that $\lambda_{n}\left(z_{A}\right)=\lambda_{A}^{+}$, from which $z_{A}=\left(n-\lambda_{A}^{+}\right) /\left(\lambda_{A}^{+}\right)^{1 / 2}$.

To prove that $z_{A}=z\left(\lambda_{A}, \alpha_{A}\right)$, it is necessary to show that $z_{A}$ satisfies (9), that is,

(i) for all $\lambda \geq \lambda_{A}, \Phi_{P}\left(\lambda, z_{A}\right) \geq \alpha_{A}$,

(ii) for $0 \leq y<z_{A}$, there exists $\tilde{\lambda} \geq \lambda_{A}$ for which $\Phi_{P}(\tilde{\lambda}, y)<\alpha_{A}$.

Consider claim (i). Since $\Phi_{P}\left(\lambda, z_{A}\right)>\Phi_{P}\left(\lambda, z_{A}\right)$ and $\Phi_{P}\left(\lambda, z_{A}\right)$ is nondecreasing in $\lambda$, it is enough to show that $\Phi_{P}\left(\lambda_{A}, z_{A}\right) \geq \alpha_{A}$. . One of the following two cases is possible: either $\lambda_{A}<\lambda_{A}^{+}$and $A \neq A^{\overline{+}}$ or $\lambda_{A}=\lambda_{A}^{+}$and $A=A^{+}$. For $\lambda_{A}<\lambda_{A}^{+}, \underline{\Phi_{P}}\left(\lambda_{A}, z_{A}\right)=\alpha_{A}$ by construction. For $\lambda_{A}=\lambda_{A}^{+}$, point $A$ lies on $C_{n-1}$ and $\underline{\Phi_{P}}\left(\lambda_{A}, z_{A}\right)>\overline{\alpha_{A}}$ because $\underline{\Phi_{P}}\left(\lambda, z_{A}\right)$ has a jump at $\lambda_{A}^{+}=\lambda_{n}\left(z_{A}\right)$.

Consider claim (ii). From Lemma 1, since $z_{A}>y$, it follows that $\lambda_{n}\left(z_{A}\right)<\lambda_{n}(y)$. In other words, the $n$th jump for $\Phi_{P}\left(\lambda, z_{A}\right)$ occurs earlier than that for $\Phi_{P}(\lambda, y)$. By construction, $\mathbb{P}\left(X_{\lambda_{n}\left(z_{A}\right)} \leq n-1\right)=\alpha_{A}$. Because $\mathbb{P}\left(X_{\lambda} \leq n-1\right)$ is continuous and decreasing in $\lambda$, there exists some $\tilde{\lambda} \in\left(\lambda_{n}\left(z_{A}\right) ; \lambda_{n}(y)\right)$ such that

$$
\Phi_{P}(\tilde{\lambda}, y)=\mathbb{P}\left(X_{\tilde{\lambda}} \leq n-1\right)<\mathbb{P}\left(X_{\lambda_{n}\left(z_{A}\right)} \leq n-1\right)=\alpha_{A}
$$

Since both claims are proved, $z_{A}=z\left(\lambda_{A}, \alpha_{A}\right)$.

(c) Clearly, the $\alpha$ th percentile of $X_{\lambda}$ is at most $\lambda+z(0, \alpha) \sqrt{\lambda}$. Using (b), for $\lambda=0, n=1$. Solving for $\mu$ in $\mathbb{P}\left(X_{\mu} \leq 0\right)=\alpha, \mu=-\log \alpha$. Then $z(0, \alpha)=(1+\log \alpha) /(\sqrt{-\log \alpha})$. This completes the proof of the corollary. 


\section{Discussion}

Theorem 1, proved in Section 2, extends the monotonicity results derived by Kane [2]. He showed in his Theorem 2.3 that $\mathbb{P}\left(\sum_{j=1}^{n}\left(X_{\lambda}\right)_{j} \geq n \lambda\right)$ is strictly decreasing in $n$, where $\left(X_{\lambda}\right)_{j}$ are independent Poisson random variables with positive integer mean $\lambda$. This result is a specific case of Theorem 1 proved in this paper for $z=0$.

The nondecreasing lower bound $\Phi_{P}(\lambda, z)$ allows the analysis of cases when the variability of the control parameter is larger than that expected from the Poisson hypothesis.

Consider the following example. A company caters an expensive perishable product to the clients of a hotel chain that is comprised of a large number of identical hotels. The daily product demand for each hotel $X_{\lambda}$ has the Poisson distribution with random demand rate $\lambda$, which is a function of the number of people who are staying in the hotel. The hotel chain informs the catering company about the number of people in each hotel for the next day. The daily demand for each hotel is independent from the demand experienced by the other hotels. The product must be delivered in the morning before the demand is known. Hotels do not share their products. As a result, at the end of the day some of the hotels may have unsatisfied demand, while others return the product to the catering company.

Service quality is measured in the following way. Every morning each hotel reports whether the previous day's demand was met to the chain management. The hotel chain requires a $95 \%$ service level, i.e. in $95 \%$ of the reports the demand should be met completely. The performance is measured on a yearly basis.

The company's manager believes that the demand rate $\lambda$ has a triangular distribution, i.e. there exists the most likely demand rate $\lambda_{M} \in\left[\lambda_{L} ; \lambda_{H}\right]$ and the values outside $\left[\lambda_{L} ; \lambda_{H}\right]$ are not credible. The company's manager wants to use a simple policy to keep the inventory level enough to meet the daily demand at $z$ standard deviations above its mean. Therefore, $z$ should be chosen so that $\lambda+z \sqrt{\lambda}$ is greater than $X_{\lambda}$ in at least $95 \%$ of cases for all $\lambda \in\left[\lambda_{L} ; \lambda_{H}\right]$.

For the triangular distribution, the expected daily hotel demand is

$$
\begin{aligned}
& \mathbb{E}(\lambda+z \sqrt{\lambda}) \\
& = \begin{cases}\frac{\lambda_{H}+\lambda_{M}+\lambda_{L}}{3} & \text { if } \lambda_{M} \in\left(\lambda_{L} ; \lambda_{H}\right), \\
+\frac{8}{15} \frac{\lambda_{L}^{5 / 2}\left(\lambda_{H}-\lambda_{M}\right)-\lambda_{M}{ }^{5 / 2}\left(\lambda_{H}-\lambda_{L}\right)+\lambda_{H}{ }^{5 / 2}\left(\lambda_{M}-\lambda_{L}\right)}{\left(\lambda_{H}-\lambda_{L}\right)\left(\lambda_{H}-\lambda_{M}\right)\left(\lambda_{M}-\lambda_{L}\right)} z & \\
\frac{1}{3}\left(\lambda_{H}+2 \lambda_{L}\right)+\frac{4}{15} \frac{2 \lambda_{H}^{3 / 2}+4 \lambda_{H} \sqrt{\lambda_{L}}+6 \sqrt{\lambda_{H}} \lambda_{L}+3 \lambda_{L}{ }^{3 / 2}}{\left(\sqrt{\lambda_{H}}+\sqrt{\lambda_{L}}\right)^{2}} & \text { if } \lambda_{M}=\lambda_{L}, \\
\frac{1}{3}\left(2 \lambda_{H}+\lambda_{L}\right)+\frac{4}{15} \frac{3 \lambda_{H}^{3 / 2}+6 \lambda_{H} \sqrt{\lambda_{L}}+4 \sqrt{\lambda_{H}} \lambda_{L}+2 \lambda_{L}{ }^{3 / 2}}{\left(\sqrt{\lambda_{H}}+\sqrt{\lambda_{L}}\right)^{2}} z & \text { if } \lambda_{M}=\lambda_{H} .\end{cases}
\end{aligned}
$$

Suppose that the normal distribution is used to approximate the demand. To provide a $95 \%$ service level, $z=\Phi^{-1}(0.95) \approx 1.645$. The normal approximation overestimates or underestimates the service level for some values of $\lambda$. If the distribution of $\lambda$ makes overestimation more probable, then the expected service level is less than $95 \%$.

To guarantee the quality level for all $\lambda \in\left[\lambda_{L} ; \lambda_{H}\right], z$ is set to be equal to $z\left(\lambda_{L}, \alpha\right)$. For instance, if $\lambda_{L}=10$ then, from Corollary $1(\mathrm{~b}), z\left(\lambda_{L}, 0.95\right) \approx 1.883$. This results in an expected service level higher than $95 \%$ because, for $\lambda>\lambda_{L}$, the daily demand is met at least in $95 \%$ of cases. 
TABLE 1: Service quality estimates (in percentages) for the demand met at the levels $\Phi^{-1}(0.95)$ and $z\left(\lambda_{L}, 0.95\right)$ standard deviations above the mean.

\begin{tabular}{cccccccccc}
\hline \multirow{2}{*}{ Level } & $\lambda_{H}$ & \multicolumn{8}{c}{$\lambda_{M}$} \\
\cline { 3 - 10 }$\Phi^{-1}(0.95)$ & 10 & 15 & 30 & 60 & 90 & 120 & 135 & 150 \\
& 15 & 94.24 & 94.31 & & & & & & \\
& 30 & 94.24 & 94.29 & 94.35 & & & & & \\
& 60 & 94.42 & 94.44 & 94.48 & 94.53 & & & & \\
& 90 & 94.38 & 94.49 & 94.61 & 94.66 & 94.66 & & & \\
& 120 & 94.60 & 94.61 & 94.53 & 94.70 & 94.67 & 94.66 & & \\
& 135 & 94.55 & 94.55 & 94.60 & 94.63 & 94.70 & 94.67 & 94.69 & \\
& 150 & 94.61 & 94.57 & 94.64 & 94.73 & 94.71 & 94.70 & 94.72 & 94.71 \\
\hline$z\left(\lambda_{L}, 0.95\right)$ & 15 & 96.32 & 96.38 & & & & & & \\
& 30 & 96.25 & 96.41 & 96.40 & & & & & \\
& 60 & 96.40 & 96.45 & 96.50 & 96.56 & & & & \\
& 90 & 96.62 & 96.54 & 96.53 & 96.63 & 96.63 & & & \\
& 120 & 96.54 & 96.59 & 96.56 & 96.70 & 96.64 & 96.68 & & \\
& 135 & 96.55 & 96.59 & 96.55 & 96.61 & 96.72 & 96.66 & 96.71 & \\
& 150 & 96.56 & 96.53 & 96.60 & 96.71 & 96.67 & 96.71 & 96.68 & 96.75 \\
\hline
\end{tabular}

The following simulation built in RISK SOLVER illustrates the service quality overestimation and underestimation effects. Service quality is estimated as the fraction of time when the daily demand is not greater than the inventory. In Table 1 we present the results for two inventory levels: $\lambda+\Phi^{-1}(0.95) \sqrt{\lambda}$ and $\lambda+z\left(\lambda_{L}, 0.95\right) \sqrt{\lambda}$. The top and the bottom halves of the table are the estimates of service quality given by $\mathbb{P}\left(X_{\lambda} \leq \lambda+\Phi^{-1}(0.95) \sqrt{\lambda}\right)$ and $\mathbb{P}\left(X_{\lambda} \leq \lambda+z\left(\lambda_{L}, 0.95\right) \sqrt{\lambda}\right)$, correspondingly.

The distribution for the daily demand $X_{\lambda}$ is set as the Poisson distribution with random demand rate $\lambda$. Table 1 summarizes the results for 35 different triangular distributions of $\lambda$. The lowest rate $\lambda_{L}$ is fixed at 10 items per day. The highest rate $\lambda_{H}$ varies from 15 to 150 items per day and the most likely rate $\lambda_{M}$ varies from $\lambda_{L}$ to $\lambda_{H} .10000$ trials are run for every parameter combination.

As can be seen in this particular example, the normal approximation results in service quality lower than $95 \%$. In this case, service under-provisioning is under $1 \%$. At the same time, the usage of $z\left(\lambda_{L}, 0.95\right)$ consistently leads to service quality higher than the target value by between $1 \%$ and $2 \%$. When the penalty for service under-provisioning is significantly higher than the cost of over-provisioning, the usage of $z\left(\lambda_{L}, \alpha\right)$ is justified.

To conclude, in this paper we presented the step function $\Phi_{P}(\lambda, z)$, a lower bound for the Poisson CDF at $z$ standard deviations above mean $\lambda$. In Theorem 1 we proved that $\Phi_{P}(\lambda, z)$ is nondecreasing in both $\lambda$ and $z$, which is an extension to the monotonicity results derived by Kane [2]. As an interesting corollary of the bound's properties, we constructed the function $z(\lambda, \alpha)$, the minimal $z$ such that, for any Poisson random variable with the mean greater or equal to $\lambda$, its $\alpha$ th percentile is at most $z$ standard deviations above its mean. As a practical application of the results, we considered an inventory management problem, where the demand has the Poisson distribution with random rate $\lambda$. We compared two policies defining the inventory level necessary to provide target service quality $\alpha$; one to keep the inventory level at $\Phi^{-1}(\alpha)$ and another to keep it at $z(\lambda, \alpha)$ standard deviations above the demand mean $\lambda$. In the simulation for this problem, the normal approximation consistently resulted in service under-provisioning, 
while $z(\lambda, \alpha)$ led to the service quality being higher than the target value. As a result, $z(\lambda, \alpha)$ is useful when overestimating the expected service quality involves high penalties. Future research could focus on exploring the tightness of the constructed lower bound $\underline{\Phi_{P}}(\lambda, z)$.

\section{Acknowledgements}

The author expresses her gratitude to her supervisor, Associate Professor E. Pinker, who offered valuable assistance and guidance. Special thanks also to the referee, whose valuable suggestions helped to streamline the proofs and paper organization significantly.

\section{References}

[1] Adell, J. A. And Jodra, P. (2005). The median of the Poisson distribution. Metrika. 61, 337-346.

[2] Kane, J. M. (2001). Monotonic approach to central limits. Proc. Amer. Math. Soc. 129, 2127-2133.

[3] Khattri, S. K. (2010). Three proofs of the inequality $e<\left(1+\frac{1}{n}\right)^{n+0.5}$. Amer. Math. Monthly 117, 273-277. 